\title{
Solid-liquid phase equilibrium of trans-cinnamic acid, $p$-coumaric acid and ferulic acid in water and organic solvents: Experimental and modelling studies
}

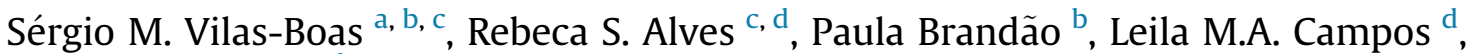 \\ João A.P. Coutinho ${ }^{\text {b }}$, Simão P. Pinho ${ }^{\text {a, }}$, Olga Ferreira ${ }^{a, c}{ }^{\text {, * }}$ \\ ${ }^{a}$ Centro de Investigação de Montanha (CIMO), Instituto Politécnico de Bragança, Campus de Santa Apolónia, 5300-253, Bragança, Portugal \\ b CICECO - Aveiro Institute of Materials, Department of Chemistry, University of Aveiro, 3810-193, Aveiro, Portugal \\ ${ }^{\mathrm{c}}$ Laboratory of Separation and Reaction Engineering - Laboratory of Catalysis and Materials (LSRE-LCM), Instituto Politécnico de Bragança, Campus de \\ Santa Apolónia, 5300-253, Bragança, Portugal \\ d Chemical Engineering Post-Graduate Program, Salvador University (UNIFACS), Salvador, BA, 40140-110, Brazil
}

\section{A R T I C L E I N F O}

Article history:

Received 12 May 2020

Received in revised form

26 June 2020

Accepted 30 June 2020

Available online 4 July 2020

\section{Keywords:}

Cinnamic acid derivatives

Solubility

Solid phase studies

NRTL-SAC model

Abraham solvation model

\begin{abstract}
A B S T R A C T
The solubility of the trans isomers of cinnamic acid, $p$-coumaric acid and ferulic acid was measured in water and seven organic solvents (methanol, ethanol, 1-propanol, 2-propanol, 2-butanone, ethyl acetate and acetonitrile), at $298.2 \mathrm{~K}$ and $313.2 \mathrm{~K}$, using the analytical shake-flask technique. The melting temperatures and enthalpies of the solutes were studied by differential scanning calorimetry, while solute solid structures were identified by powder and single X-ray diffraction.

The NRTL-SAC model was applied to calculate the solubility of trans-cinnamic acid and trans-ferulic acid in pure solvents. For trans-p-coumaric acid, the NRTL-SAC was combined with the Reference Solvent Approach, as the solute melting properties could not be determined. The global average relative deviations (ARD) were $32 \%$ and $41 \%$, in the correlation and prediction stages, respectively. The Abraham solvation model was also applied. The global ARD were $20 \%$ for correlation and $29 \%$ for predictions, which can be considered very satisfactory results for these semi-predictive models.
\end{abstract}

(C) 2020 Elsevier B.V. All rights reserved.

\section{Introduction}

Naturally occurring phenolic acids are well studied for their bioactive properties and present a wide distribution in plant material, where they can be found in the free form, or conjugated to other molecules [1]. Among this family of compounds, two major classes can be distinguished based on their structure: benzoic acid derivatives and cinnamic acid derivatives [1,2]. In general, cinnamic acid derivatives are more abundant in nature, especially ferulic acid, caffeic acid, $p$-coumaric acid and sinapic acid [2-4], having many applications in the pharmaceutical, food, and cosmetic industries due to their chemical and biological properties [2,3].

The main aim of this work is to study the solubility of the trans isomers (for simplicity, the prefix trans will be omitted in the text)

\footnotetext{
* Corresponding author. Centro de Investigação de Montanha (CIMO), Instituto Politécnico de Bragança, Campus de Santa Apolónia, 5300-253, Bragança, Portugal. E-mail address: oferreira@ipb.pt (O. Ferreira).
}

of cinnamic acid and two derivatives ( $p$-coumaric acid and ferulic acid) in water and seven pure organic solvents (methanol, ethanol, 1-propanol, 2-propanol, 2-butanone, ethyl acetate and acetonitrile) at $298.2 \mathrm{~K}$ and $313.2 \mathrm{~K}$. Whenever possible, the solubility data were critically compared to literature. Some solubility studies can be found for cinnamic acid [5-8], p-coumaric acid [9,10], and ferulic acid [5,11-15], but for several binary systems, the solubility data are reported for the first time.

The structures of the solutes are shown in Fig. 1. As can be seen, relatively to the simplest cinnamic acid (3-phenylacrylic acid), $p$ coumaric acid (3-(4-hydroxyphenyl)acrylic acid) has an additional hydroxyl group and ferulic acid (3-(4-hydroxy-3-methoxyphenyl) acrylic acid) presents a hydroxyl and a methoxy group.

The trans isomer is the predominant form of cinnamic acid, being Chinese cinnamon a major natural source, and presents relevant pharmaceutical and biological properties, such as antibacterial, antiinflammatory, antifungal, antioxidant and antitumor activities [16]. Cinnamic acid is also an ingredient used in several personal-care products and non-cosmetic products [17]. Similarly, $p$-coumaric 
<smiles>O=C(O)/C=C/c1ccccc1</smiles>

(a)<smiles>O=C(O)/C=C/c1ccc(O)cc1</smiles>

(b)<smiles>COc1cc(/C=C/C(=O)O)ccc1O</smiles>

(c)

Fig. 1. Chemical structures of the trans isomers of: (a) cinnamic acid; (b) p-coumaric acid and (c) ferulic acid.

acid and its conjugates also present bioactive properties in different dimensions, such as antioxidant, anti-tumour, antimicrobial, antivirus, anti-inflammatory, antiplatelet aggregation, anxiolytic, antipyretic, analgesic, and anti-arthritis [18]. Also, this compound has been identified as a competitive inhibitor of tyrosinase, and studied as a potential skin-lightening cosmetic ingredient [19]. Finally, ferulic acid is the most abundant phenolic acid found in cereal grains, and in different vegetables and fruits, such as citrus fruits, banana, coffee, eggplant, bamboo shoots, beetroot, cabbage, spinach, and broccoli $[2,20]$. It presents antioxidant, antimicrobial, anti-inflammatory, anti-thrombosis, and anti-tumour activities, among others. It is widely used as an ingredient in the food and cosmetic areas and as a raw material for the production of other important compounds, such as vanillin, sinapic acid and curcumin [20,21]. Thus, for the adequate design of products and processes it is extremely relevant to know some of their physicochemical properties, namely reliable solubility data in pure and mixed solvents, as the compounds are in the solid state at room conditions.

Finally, to model the solid-liquid equilibria data, two semipredictive thermodynamic models were selected: (1) the semipredictive Nonrandom Two-Liquid Segment Activity Coefficient (NRTL-SAC) model proposed by Chen and Song [22] that was already used to describe the solubility of phenolic compounds in water and organic solvents [23-28]; (2) the Abraham solvation model [29-31] that has been applied to calculate the solubility of benzoic acid derivatives [28,32-39], and cinnamic acid derivatives $[8,40]$. To support the description of the solid-liquid equilibria, the melting properties of the pure solutes were measured by Differential Scanning Calorimetry (DSC) and solid phase studies were carried out by X-Ray Diffraction (XRD).

\section{Experimental}

\subsection{Chemicals}

Ultrapure water (resistivity of $18.2 \mathrm{M} \Omega \mathrm{cm}$, free particles $\geq 0.22 \mu \mathrm{m}$ and total organic carbon $<5 \mu \mathrm{g} \mathrm{dm}^{-3}$ ) was used. All the organic compounds were used as received from the suppliers and are listed in Table 1. The solids were kept in a desiccator to avoid water contamination.

Table 1

Mass purity (\%), CAS number and source of the organic compounds used in this work.

\begin{tabular}{llll}
\hline Compound & Mass purity (\%) & CAS number & Source \\
\hline trans-cinnamic acid & $\geq 99.5$ & $140-10-3$ & Alfa Aesar \\
p-coumaric acid & $\geq 99.9$ & $7400-08-0$ & Merck KGaA \\
trans-ferulic acid & $\geq 99.9$ & $537-73-5$ & Alfa Aesar \\
methanol & $\geq 99.9$ & $67-56-1$ & Carlo Erba \\
ethanol & $\geq 99.9$ & $64-17-5$ & Carlo Erba \\
1-propanol & $\geq 99.5$ & $71-23-8$ & Carlo Erba \\
2-propanol & $\geq 99.9$ & $67-63-0$ & Honeywell \\
2-butanone & $\geq 99.5$ & $78-93-3$ & Sigma-Aldrich \\
ethyl acetate & $\geq 99.9$ & $141-78-6$ & Carlo Erba \\
acetonitrile & $\geq 99.9$ & $75-05-8$ & Sigma-Aldrich \\
\hline
\end{tabular}

a The purity was obtained in the certificate of analysis issued by the manufacturer.

\subsection{Melting properties}

The melting temperatures and enthalpies were determined by DSC (model 204 F1 Phoenix, NETZSCH) using a nitrogen flowing system. Samples of $3-8 \mathrm{mg}( \pm 0.1 \mathrm{mg}$ ) were hermetically sealed into aluminum crucibles. The heating and cooling rates were $1 \mathrm{~K} /$ min and $2 \mathrm{~K} / \mathrm{min}$, respectively. The experiments were performed from $293.2 \mathrm{~K}$ to $523.2 \mathrm{~K}$ for cinnamic acid, from $293.2 \mathrm{~K}$ to $503.2 \mathrm{~K}$ for $p$-coumaric acid, and from $293.2 \mathrm{~K}$ to $473.2 \mathrm{~K}$ for ferulic acid. At least three runs were considered to calculate the final average results. An external calibration was performed using 11 compounds (water, 4-nitrotoluene, naphthalene, benzoic acid, diphenyl acetic acid, indium, anthracene, tin, caffeine, bismuth and zinc). The onset value was considered as the melting temperature.

\subsection{Solubility experiments}

The solubility experiments were carried out by the isothermal shake-flask method, which was described in detail elsewhere [27,41]. In summary, around $80 \mathrm{ml}$ of a saturated solution of each binary system was prepared and placed in a thermostatic bath (maximum temperature deviation of $\pm 0.1 \mathrm{~K}$ ). From preliminary experiments, the optimum stirring and settling times were found to be $32 \mathrm{~h}$ and $15 \mathrm{~h}$, respectively. After reaching equilibrium, three samples of around $0.3 \mathrm{~cm}^{3}$ were collected from the supernatant solution, using pre-heated plastic syringes coupled to a polypropylene filter $(0.45 \mu \mathrm{m}$ pore size $)$.

In previous works $[27,28,41]$, the composition of the samples was quantified by gravimetry. However, tests showed that transcinnamic acid was thermally unstable, once it presented a weight loss of $6.3 \%$, after remaining at $343.2 \mathrm{~K}$ for one week inside an oven, and a weight loss of $1.3 \%$ after one month at $303.2 \mathrm{~K}$ (along with significant color change in both cases). Therefore, the selected analytical method was UV-Vis spectroscopy (model T70, PG Instruments), at wavelengths $273 \mathrm{~nm}$ (cinnamic acid), $310 \mathrm{~nm}$ ( $p$ coumaric acid) and $321 \mathrm{~nm}$ (ferulic acid). The samples were diluted in a mixture of water + ethanol (proportion 35:65 by wt.\%), placed in cuvettes ( $5 \mathrm{~mm}$ optical path) and then read at least three times. The calibration curves $\left(\mathrm{R}^{2} \geq 0.998\right)$ were obtained using seven standard solutions.

\subsection{Solid-phase studies}

\subsubsection{Samples}

The solid phase of the aromatic acids, as received from suppliers and crystallized after evaporation of a set of selected solvents, was analyzed by powder or single crystal X-Ray diffraction.

\subsubsection{Powder and single X-ray diffraction}

Powder XRD data were collected on a X'Pert MPD Philips diffractometer, using Cu-Ka radiation $(\lambda=1.5406 \AA)$, with a curved graphite monochromator, a set incident area of $10 \mathrm{~mm}^{2}$, and a flat plate sample holder, in a Bragg-Brentano para-focusing optics configuration. Intensity data were collected by the step counting 
method (step $0.02^{\circ}$ and time $5 \mathrm{~s}$ ) in the range $5^{\circ}<2 \theta<50^{\circ}$.

The cell parameters of suitable crystals of the solutes provided from suppliers as well the solid samples obtained after evaporating the solvent (water, methanol, ethanol, 2-butanone, ethyl acetate and acetonitrile) were determined on a Bruker D8 Quest photon 100 CMOS, with monochromated Mo-K $\alpha$ radiation $(\lambda=0.71073 \AA$ ) and operating at 150 (2) K. The selected crystals were placed at $40 \mathrm{~mm}$ from the detector and the spots were measured using different counting times (varying from 10 to $30 \mathrm{~s}$ ).

\section{Thermodynamic modeling}

\subsection{The NRTL-SAC model}

The NRTL-SAC model was already applied in previous studies [22-25,28,41-43], and is described in detail elsewhere [22,42]. The model describes each molecule using four conceptual segments related to the different surface characteristics: hydrophobic $(\mathrm{X})$, hydrophilic $(Z)$, polar attractive $\left(Y_{+}\right)$, and polar repulsive $\left(Y_{-}\right)$. These parameters were reported for a large number of solvents, including those studied in this work [22,42]. Therefore, only the molecular descriptors of the solute need to be estimated.

Assuming pure solid phase and neglecting the heat capacity change upon melting which has often a small impact in equilibrium calculations, the solubility of a solid solute in a liquid solvent can be calculated from the equation [44]:

$\ln x_{s}=\frac{\Delta_{m} H}{R T_{m}}\left(1-\frac{T_{m}}{T}\right)-\ln \gamma_{s}$

where $\boldsymbol{x}_{\boldsymbol{s}}$ is the mole fraction solubility of the solute $S, R$ is the ideal gas constant, $T$ is the absolute temperature, $T_{m}$ is the absolute melting temperature of the solute, $\Delta_{m} \boldsymbol{H}$ its melting enthalpy, and $\gamma_{s}$ is the activity coefficient of the solute $S$ in the binary liquid solution, here calculated using the NRTL-SAC model [22].

As can be seen from Eq. (1), accurate melting properties are needed to predict the solubility data. Alternatively, the NRTL-SAC model can be combined with the Reference Solvent Approach (RSA), proposed by Abildskov and O'Connell $[45,46]$. In this methodology, the use of a reference solvent eliminates the need of the melting properties, being a useful tool whenever the melting properties present high uncertainties or are unavailable. Briefly, the RSA can be described by:

$\ln x_{S i}=\ln x_{S j}+\ln \gamma_{S j}\left(T,\left\{x_{S}\right\}_{j}\right)-\ln \gamma_{S i}\left(T,\left\{x_{S}\right\}_{i}\right)$

where $x_{S i}$ is the mole fraction solubility of solute $S$ in a solvent $i, x_{S j}$ is the solubility of the same solute in a reference solvent $j$, $\gamma_{S i}\left(T,\left\{x_{S}\right\}_{i}\right)$ is the activity coefficient of the solute in solvent $i$, while $\gamma_{S j}\left(T,\left\{x_{S}\right\}_{j}\right)$ is the activity coefficient of the solute in the reference solvent $j$.

As can be seen in Eq. (2), the experimental solubility of a solute in a given reference solvent is used along with the activity coefficients calculated by the NRTL-SAC model. For a given set of data, the optimal reference solvent is found by:

$\min _{j}\left|\sum_{i=\text { data }} \delta \ln x_{S, i j}\right|=\min _{j}\left|\sum_{i=\text { data }}\left(\ln x_{S i}+\ln \gamma_{S i}\right)-N\left(\ln x_{S j}+\ln \gamma_{S j}\right)\right|$

where $\sum_{i=\text { data }} \delta \ln x_{S, i j}$ is the error associated to the mole fraction solubilities of solute $S$ in all the solvents assuming a reference solvent $j$, and $N$ is the number of experimental data points in a given set.

\subsection{The Abraham solvation model}

The partition coefficient between water and a solvent $\left(\boldsymbol{P}_{\boldsymbol{S}}\right)$ can be approximated by the ratio of the molar solubilities of a solute in the organic solvent $\left(\boldsymbol{S}_{\boldsymbol{s}}\right)$ and in water $\left(\boldsymbol{S}_{\boldsymbol{w}}\right)$ :

$P_{S}=\frac{S_{S}}{S_{w}}$

As discussed by Abraham and co-authors [31], Eq. (4) only holds if: (a) the solid phase in equilibrium with both solvents is the same; (b) the secondary medium activity coefficient of the solute in the two phases is near unity; the same (undissociated, if ionizable) chemical species should be present in each phase. In addition, the partition of a solute between two fluid phases can be correlated by two linear free energy relationships (LFERs) [29-31].

$\log \left(P_{S}\right)=c+e E+s S+a A+b B+v V$

$\log \left(K_{S}\right)=c+e E+s S+a A+b B+l L$

Eq. (5) calculates the solute partition between two condensed phases and, Eq. (6), the partition between a gas phase and an organic solvent. In those equations, the uppercase descriptors $(E, S$, $A, B, V$ and $L$ ) represent the Abraham solute descriptors, where $E$ is the solute excess molar refractivity, $S$ refers to the solute dipolarity/ polarizability, $A$ and $B$ account for the overall solute hydrogen bond acidity and basicity, $V$ is the solute's McGowan characteristic molecular volume and $L$ is the logarithm of the gas-to-hexadecane partition coefficient at $298.15 \mathrm{~K}$. The lowercase regression coefficients and constants represent condensed phase properties, already available for a large number of solvents. For each solute, $V$ can be calculated from its molecular structure. The descriptor $E$ can be calculated from the solute's refractive index, which can be experimentally obtained or, if unavailable, estimated using ACD free software.

For the solvents studied in this work, these coefficients have already been reported in literature [31]. Regarding the solute descriptors, they can be estimated by multiple linear regression, using experimental solubility data. Estimations of the solute descriptors have been already reported for the monomeric and dimeric forms of trans-cinnamic acid [8] and for the monomeric form of p-coumaric acid [40], but no information was found for ferulic acid.

\section{Results and discussion}

\subsection{Melting properties}

The melting temperatures and enthalpies obtained in this work are presented in Table 2 along with the data found in literature [5,6,9,10,14,47-52]. In Fig. S1 of Supporting Information (SI), exemplificative thermograms of trans-cinnamic acid and ferulic acid are presented.

The melting properties of $p$-coumaric acid could not be derived from the DSC experiments performed in this work due to decomposition of the samples upon melting. Several experiments were performed, always showing an exothermic transition immediately after an endothermic one, precluding the correct integration of the melting peak. The melting temperatures reported by Alevizou et al. [9] and Ji et al. [10] are similar, whereas the melting enthalpies present larger deviations.

In the case of trans-cinnamic acid, the melting temperature $(406.9 \pm 0.2 \mathrm{~K})$ and enthalpy $\left(22.1 \pm 0.2 \mathrm{~kJ} \mathrm{~mol}^{-1}\right)$ obtained in this work are consistent with the average literature data $\left(T_{\mathrm{m}}=405.7 \pm 0.7 \mathrm{~K}\right.$ and $\left.\Delta_{\mathrm{m}} H=23.1 \pm 1.5 \mathrm{~kJ} \mathrm{~mol}^{-1}\right)$. For ferulic acid, the melting temperature obtained in this work $(445.8 \pm 0.2 \mathrm{~K})$ is 
Table 2

Comparison of the melting temperatures and enthalpies of the studied aromatic acids found in the literature and measured in this work.

\begin{tabular}{|c|c|c|c|c|}
\hline Compound & $T_{\mathrm{m}} / \mathrm{K}$ & $\Delta_{\mathrm{m}} H / \mathrm{kJ} \cdot \mathrm{mol}^{-1}$ & Methodology & Reference \\
\hline \multirow[t]{6}{*}{ trans-cinnamic acid } & 406.1. \pm 0.4 & $22.2 \pm 0.8$ & DSC & [5] \\
\hline & $406.2 \pm 0.3$ & $22.2 \pm 0.4$ & DSC & [6] \\
\hline & 406.2 & 22.6 & $\mathrm{NA}^{\mathrm{a}}$ & [48] \\
\hline & 405.5 & 25.7 & DSC & [49] \\
\hline & 404.8 & 22.6 & DSC & [50] \\
\hline & $406.9 \pm 0.2^{\mathrm{b}}$ & $22.1 \pm 0.1$ & DSC & this work \\
\hline \multirow[t]{3}{*}{ p-coumaric acid } & $492.4 \pm 0.3$ & $27.4 \pm 0.9$ & DSC & [9] \\
\hline & $494.4 \pm 0.2$ & $34.3 \pm 0.02$ & DSC & [10] \\
\hline & $--^{c}$ & $--^{c}$ & DSC & this work \\
\hline \multirow[t]{7}{*}{ ferulic acid } & $444.6 \pm .0 .5$ & $33.3 \pm 1.2$ & DSC & [5] \\
\hline & $448.0^{\mathrm{d}}$ & $33.5^{\mathrm{d}}$ & DSC & [14] \\
\hline & $447.7^{e}$ & $36.3^{e}$ & & \\
\hline & $445.9 \pm 0.5$ & $34.7 \pm 0.2$ & DSC & [47] \\
\hline & $444.9 \pm 0.4$ & $31.9 \pm 0.9$ & DSC & [51] \\
\hline & $445.1 \pm 0.9$ & $33.5 \pm 0.5$ & DSC & [52] \\
\hline & $445.8 \pm 0.2^{\mathrm{b}}$ & $38.4 \pm 0.2$ & DSC & this work \\
\hline
\end{tabular}

\footnotetext{
a Not available.

b The experimental onset temperatures were considered as melting temperatures, in this work.

c Decomposition upon melting.

d Melting properties of ferulic acid as received from the supplier.

e Melting properties of ferulic acid recovered from an aqueous saturated solution (after evaporation of the solvent).
}

very close to the average melting temperature calculated from literature $(445.7 \pm 1.2 \mathrm{~K})$, being closer to the value reported by Emel'yanenko et al. [47]. The melting enthalpy $\left(38.4 \pm 0.2 \mathrm{~kJ} \mathrm{~mol}^{-1}\right)$, however, is higher than the literature average $\left(\Delta_{\mathrm{m}} \mathrm{H}=33.9 \pm 1.5 \mathrm{~kJ} \mathrm{~mol}^{-1}\right)$. Shakeel et al. [14] measured the melting properties for ferulic acid obtained directly from the supplier and recovered from saturated aqueous solutions, being the later much closer to the melting enthalpy obtained in this work. In fact, the melting temperatures reported by the authors [14] for both cases are very close, indicating that no solid phase transformation occurred in the solid recovered from the aqueous mixtures. For this reason, both data were included in Table 2 .

For trans-cinnamic acid and ferulic acid, in the studied temperature range, only the melting phase transition peak was identified in the thermograms. The melting peak shapes of transcinnamic acid presented modifications over successive runs (at heating rates of $1 \mathrm{~K} / \mathrm{min}$ and cooling rates of $2 \mathrm{~K} / \mathrm{min}$ ) leading generally to lower temperature and enthalpy values (as shown in Fig. S2 of SI). The samples of ferulic acid could not be crystalized after melting. Therefore, only independent first runs were considered.

\subsection{Experimental solubilities}

The solubilities of the trans isomers of cinnamic acid, $p$-coumaric acid and ferulic acid in methanol, ethanol, 1-propanol, 2propanol, 2-butanone, ethyl acetate, acetonitrile and water at $298.2 \mathrm{~K}$ and $313.2 \mathrm{~K}$ are presented in Table 3.

Each reported data point is the average of three samples. The consistency of the measurements is confirmed by the low coefficients of variation, lower than $5.5 \%$.

In all cases, the solubility increases as the temperature increases, being larger in alcohols and 2-butanone and considerably smaller in water. This behavior was observed in previous works with benzoic acid derivatives [27,28]. Among the solutes studied, transcinnamic acid is the most soluble solute in all the organic solvents, whereas ferulic acid and $p$-coumaric acid have similar solubilities.
The latter are more soluble in water than cinnamic acid.

Trans-cinnamic acid presents the lowest melting properties compared to the other two acids (discussed in Section 4.1), meaning it has the highest ideal solubility. This effect seems to dominate over the solute-polar solvent interactions that could be higher in the cases of $p$-coumaric acid and ferulic acid due to the presence of an additional polar hydroxyl group, which certainly contributes for their slightly higher (mole fraction) solubility in water.

In the case of systems containing alcohols, the solubilities follow the order: trans-cinnamic-acid > p-coumaric acid > ferulic acid. Also, the solubility decreases as the alkyl chain length of the alcohol increases. The increase in the solubility of $p$-coumaric acid in ethanol from $298.2 \mathrm{~K}$ to $313.2 \mathrm{~K}(2.7 \%)$ is surprisingly much lower than the average increase of the solubilities of the solutes in alcohols (26.4\%), but also of the other solutes in ethanol.

For ethyl acetate, 2-butanone and acetonitrile, trans-cinnamic acid is also the most soluble solute, but ferulic acid presents higher solubilities than $p$-coumaric acid. In this case, the presence of the methoxy group seems to increase the interactions of ferulic acid with the polar non-associative solvents.

\subsection{Comparison with literature data}

The solubility data obtained in this work was critically compared to literature data [5-15,53]. In Fig. 2, a comparison between the solubility data in ethanol and water is presented, while other relevant comparisons are given in Table S1 and Figs. S3-S5 in Supporting Information.

In general, the solubilities obtained in this work are in good agreement with the literature data. For example, the solubilities in ethanol or water have ARD (calculated as the ratio between the absolute value of the difference between the solubility data obtained in this work and the average value from literature, divided by the average value from literature) lower than $13 \%$ for ethanol and lower than $5 \%$ for water, with the exception of the system $p$ coumaric acid + ethanol at $313.15 \mathrm{~K}$, where the solubility obtained in this work is $4 \mathrm{~g} / 100 \mathrm{~g}$ of solvent (ARD of $17 \%$ ) lower than the value reported by Ji et al. [10]. The solubility of ferulic acid in water reported by Noubigh et al. [53] is much higher than all the other data found in literature [5,11-15], and were not included in Fig. 2 for practical viewing purposes.

A point deserving attention is the slope of the solubility change with temperature (Fig. 2b) in the systems p-coumaric acid + alcohol (ethanol, 1-propanol, 2-propanol), in Fig. 2b, S4b and S4c. Ji and co-authors [10] employed the gravimetric method for the quantification analysis of the saturated solution, while the UV-Vis spectroscopy was preferred in this work. As discussed by Königsberger [54], several parameters, from compound purities to the analytical methods influence the final solubility value. To check the accuracy of the solubility values obtained in this work, the experiments were repeated for $p$-coumaric acid in alcohols, at both temperatures, but no significant changes were observed.

\subsection{Solid phase studies}

The solid phase of the aromatic acids, as received from suppliers as well as crystallized after evaporation of a set of selected solvents, was analyzed by powder or single crystal X-ray diffraction. It was found that, for all solutes, the solid phase recovered from evaporating the solvent kept the same structure when compared to that of the supplier.

The trans-cinnamic acid obtained from the supplier had very small crystals that were analyzed by powder X-ray diffraction, showing a pattern comparable to that published in CCDC database with number 705511 (Fig. S6). The solid phases of trans-cinnamic 
Table 3

Experimental solubility ( $\mathrm{g}$ of solute/100 $\mathrm{g}$ of solvent) of the studied cinnamic acids in water and organic solvents at $298.2 \mathrm{~K}$ and $313.2 \mathrm{~K}{ }^{\mathrm{a}, \mathrm{b}}$.

\begin{tabular}{|c|c|c|c|c|c|c|}
\hline \multirow[t]{2}{*}{ Solvent } & \multicolumn{2}{|c|}{ trans-cinnamic acid } & \multicolumn{2}{|c|}{$p$-coumaric acid } & \multicolumn{2}{|l|}{ ferulic acid } \\
\hline & $298.2 \mathrm{~K}$ & $313.2 \mathrm{~K}$ & $298.2 \mathrm{~K}$ & $313.2 \mathrm{~K}$ & $298.2 \mathrm{~K}$ & $313.2 \mathrm{~K}$ \\
\hline methanol & $32.94 \pm 0.27$ & $45.70 \pm 0.07$ & $22.79 \pm 0.27$ & $31.39 \pm 0.18$ & $21.45 \pm 0.81$ & $30.18 \pm 0.12$ \\
\hline ethanol & $25.86 \pm 0.85$ & $36.47 \pm 0.08$ & $18.78 \pm 0.84$ & $19.29 \pm 0.64$ & $11.60 \pm 0.03$ & $17.56 \pm 0.02$ \\
\hline 1-propanol & $19.29 \pm 0.68$ & $28.47 \pm 0.53$ & $10.81 \pm 0.22$ & $11.57 \pm 0.25$ & $5.82 \pm 0.16$ & $9.22 \pm 0.29$ \\
\hline 2-propanol & $16.79 \pm 0.55$ & $29.18 \pm 0.62$ & $9.44 \pm 0.26$ & $10.56 \pm 0.29$ & $6.20 \pm 0.05$ & $9.92 \pm 0.16$ \\
\hline 2-butanone & $22.46 \pm 0.15$ & $31.93 \pm 1.00$ & $8.39 \pm 0.28$ & $10.19 \pm 0.37$ & $8.41 \pm 0.16$ & $11.74 \pm 0.51$ \\
\hline ethyl acetate & $13.05 \pm 0.24$ & $19.86 \pm 0.27$ & $1.97 \pm 0.11$ & $2.85 \pm 0.03$ & $2.91 \pm 0.08$ & $4.49 \pm 0.01$ \\
\hline acetonitrile & $6.26 \pm 0.01$ & $11.42 \pm 0.03$ & $1.20 \pm 0.03$ & $2.02 \pm 0.04$ & $2.19 \pm 0.03$ & $3.85 \pm 0.06$ \\
\hline water & $0.042 \pm 0.001$ & $0.083 \pm 0.001$ & $0.056 \pm 0.001$ & $0.133 \pm 0.003$ & $0.060 \pm 0.001$ & $0.126 \pm 0.003$ \\
\hline
\end{tabular}

a Temperature and pressure standard uncertainties are $u(T)=0.10 \mathrm{~K}$ and $\mathrm{u}_{\mathrm{r}}(\mathrm{p})=0.05$, respectively.

b Standard deviations are placed after plus-minus sign.
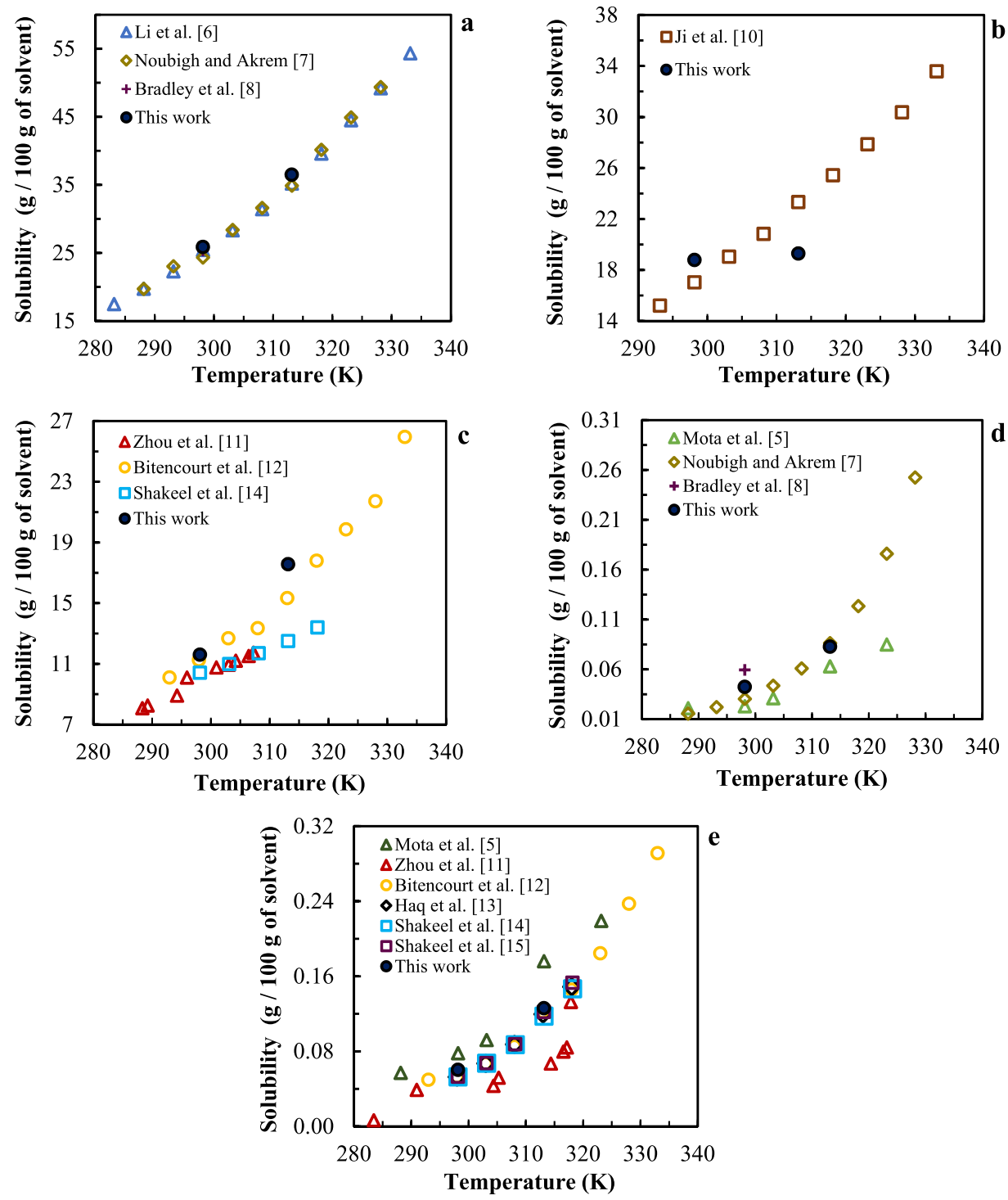

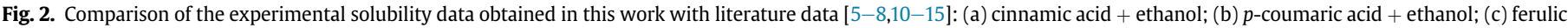
acid + ethanol; (d) cinnamic acid + water; (e) ferulic acid + water.

acid obtained after evaporation from water, ethanol, methanol, ethyl acetate, 2-butanone and acetonitrile solutions showed suitable crystals to be analyzed by single crystal X-ray diffraction. All solids crystalized in monoclinic system $P$ with the cell parameters $\mathrm{a}=5.57 \AA, \mathrm{b}=17.51 \AA, \mathrm{c}=7.61 \AA$ and $\beta=96.35^{\circ}$, which are comparable to trans-cinnamic acid published with CCDC number 705511.

Crystals of $p$-coumaric acid from supplier and obtained after evaporation from water, methanol, ethyl acetate, 2-butanone and acetonitrile solutions were indexed by single crystal X-ray 
diffraction with the following cell parameters: $a=8.70 \AA$, $\mathrm{b}=5.22 \AA, \mathrm{c}=17.06 \AA$ and $\beta=100.43^{\circ}$, monoclinic $\mathrm{P}$, which are comparable to $p$-coumaric acid published with CCDC number 945006. Additionally, all samples were analyzed by powder X-ray diffraction showing similar powder patterns. Fig. S7 compares the powder pattern of $p$-coumaric acid from supplier and the powder pattern simulated from the single crystal data of the sample published in CCDC database with number 945006.

The crystals of trans-ferulic acid from supplier, and obtained after evaporation from water, methanol, ethanol, ethyl acetate and 2-butanone solutions, crystallized in the monoclinic system $P$ with the cell parameters $\mathrm{a}=4.61 \AA, \mathrm{b}=16.76 \AA$, $\mathrm{c}=11.85 \AA$ and $\beta=91.55^{\circ}$, comparable to trans-ferulic acid deposited in CCDC database with number 950899. The structure of the bulk samples was analyzed by powder X-ray diffraction showing all the same powder pattern as the trans-ferulic acid sample with CCDC number of 950899 (Fig. S8).

\subsection{Thermodynamic modelling}

\subsubsection{NRTL-SAC and NRTL-SAC + RSA}

The optimization of the NRTL-SAC segment descriptors of the solutes was carried out using the routine Isqnonlin (MATLAB software version R2018a). The following objective function was minimized using a nonlinear least-squares method:

$F=\sum_{i}\left(\frac{\left|x_{i}^{e x p}-x_{i}^{\text {calc }}\right|}{x_{i}^{e x p}}\right)$

where $x_{i}$ is the mole fraction solubility in the solvent $i$ and the superscripts "calc" and "exp" mean the calculated and experimental values, respectively.

To assure that the NRTL-SAC parameters provide a good predictive character to the model it is advisable to include, in the correlation step, solubility data obtained in solvents with different surface characteristics. Therefore, the experimental solubilities measured in seven solvents (water, methanol, ethanol, 2-propanol, ethyl acetate, acetonitrile and 2-butanone) were used to correlate the four conceptual segment parameters $\left(X, Y^{-}, Y^{+}, Z\right)$ for each solute. Afterwards, the solubility in 1-propanol (data from this work) and in other organic solvents $[6,8,10,11,14]$ was predicted. The deviations between the experimental and calculated data were calculated as:

$A R D(\%)=\frac{1}{N P} \sum_{i} \frac{\left|x_{i}^{\text {exp }}-x_{i}^{\text {calc }}\right|}{x_{i}^{\exp }} * 100$

where $N P$ is the number of data points.

As discussed before, the melting properties obtained for transcinnamic acid and trans-ferulic acid are in satisfactory agreement with literature. For these two solutes, the NRTL-SAC model could be directly combined with Eq. (1). Nevertheless, the melting temperature and enthalpy could not be determined in this work for $p$ coumaric acid, and high uncertainties are observed in the literature data, especially for the melting enthalpies. For that reason, the RSA proposed by Abildskov and O'Connell $[45,46]$ was in this case combined with the NRTL-SAC model.

The molecular descriptors of the solutes obtained using both correlation approaches (NRTL-SAC or NRTL-SAC + RSA), the outlier solvent (presenting the highest ARD) and the global ARD are presented in Table 4.

The results above show that both NRTL-SAC and NRTLSAC + RSA approaches adequately describe the solubilities of the studied compounds, presenting ARDs varying from $23 \%$ to $38 \%$, which are satisfactory for this semi-predictive model and close to values obtained previously for similar binary systems [23,24,27,28]. Attempts to estimate the NRTL-SAC segment descriptors of transcinnamic and ferulic acids using the RSA were performed, but no significant improvements in the global ARDs were accomplished. After, the models were applied to predict the solubility in other solvents at $298.2 \mathrm{~K}$ and $313.2 \mathrm{~K}( \pm 1 \mathrm{~K})$. Those systems are shown in Table S2 of SI. A complete overview of the correlation and prediction results, using either the NRTL-SAC or NRTL-SAC + RSA, is presented in Fig. 3.

As can be seen in Fig. 3, a very good description is achieved for aqueous systems (maximum ARDs of $25 \%$ ). The model also satisfactorily correlates the solubilities in acetonitrile (ARD of 30\%) and alcohols in general (global ARD of 26\%). The highest ARDs are obtained for the systems containing ethyl acetate and 2-butanone (ARDs of $45 \%$ and $60 \%$, respectively). In general, the model provides satisfactory predictions for systems including alcohols, ethers, esters and ketones. The global ARDs for trans-cinnamic acid, p-coumaric acid and ferulic acid are $40 \%, 40 \%$ and $44 \%$, respectively. The worse predictions were found for the solubility in dimethyl sulfoxide, ethylene glycol, and methyl acetate, and generally, when deviations are more significant, the model underestimates the solubility values.

\subsubsection{Abraham solvation model}

The solute descriptors $A, B$ and $S$ were obtained by multiple linear regression using solid-liquid equilibrium data only (Eq. (5)). From our previous experience with benzoic acid derivatives [27,28], the robustness of the parameters relies in the number and diversity of systems included in the correlation. For instance, the inclusion of experimental solubility data in dimethylformamide (DMF) in the correlation set, considerably improved the overall predictions [28]. Therefore, data reported by Bradley et al. [8] and Shakeel et al. [14] for the solubility of trans-cinnamic acid and ferulic acid in another polar aprotic solvent, dimethyl sulfoxide (DMSO), were added to the same correlation set already used with the NRTL-SAC model. In the Abraham solvation model, the data are expressed in molar concentration (Eq. (4)), so all the experimental solubilities were converted from mole fraction $\left(x_{S}^{\exp }\right)$ to molar solubilities $\left(S_{S}^{\exp }\right)$ (Table S3 of SI). A global ARD of $20 \%$ was obtained in the correlation step. The model parameters, the outlier solvent and the ARD\% are shown in Table 5.

Like in a previous work [27,28], the results obtained in the correlation show an excellent description of the SLE. To the best of our knowledge, the Abraham solvation descriptors are reported for the first time for ferulic acid. Bradley et al. [8] and Acree et al. [40] have already applied the model to describe the SLE of the monomeric and dimeric forms of trans-cinnamic acid and the monomeric form of $p$-coumaric acid, respectively. In the first case, the authors considered the solubility data of trans-cinnamic acid in water and in 21 organic solvents along with water-solvent partition coefficient data of five systems. The solute parameters reported for the monomeric form [8] are very close to the parameters found in this work, being the largest deviation observed for the $E$ parameter (1.140 compared to 1.301 obtained in this work). The authors estimated $E$ through a group contribution model proposed by Platts et al. [56], which allowed the calculation of different values for both monomeric and dimeric forms of the cinnamic acid, which is different from the methodology used in this work. It is relevant to mention that, as discussed by Bradley et al. [8] and Acree et al. [40], cinnamic acids might dimerize in less polar solvents, such as alkyl benzenes, chloroalkanes and nonpolar hydrocarbons. As the set of solvents selected to correlate the parameters of solutes in both NRTL-SAC and Abraham models are composed by polar solvents, 
Table 4

NRTL-SAC estimated parameters, outlier solvent and ARD (\%) for each solute.

\begin{tabular}{|c|c|c|c|c|c|c|c|}
\hline Compound & $\mathrm{X}$ & Y- & $\mathrm{Y}+$ & $\mathrm{Z}$ & Model & Outliers & $\operatorname{ARD}(\%)$ \\
\hline trans-cinnamic acid & 0.708 & 0.000 & 0.000 & 0.524 & NRTL-SAC & 2-butanone & 23 \\
\hline$p$-coumaric acid & 0.702 & 0.023 & 0.000 & 1.702 & NRTL-SAC + RSA $^{\mathrm{a}}$ & ethyl acetate/2-butanone & 38 \\
\hline ferulic acid & 0.456 & 0.816 & 0.583 & 0.000 & NRTL-SAC & methanol & 36 \\
\hline
\end{tabular}

a The reference solvent is acetonitrile.
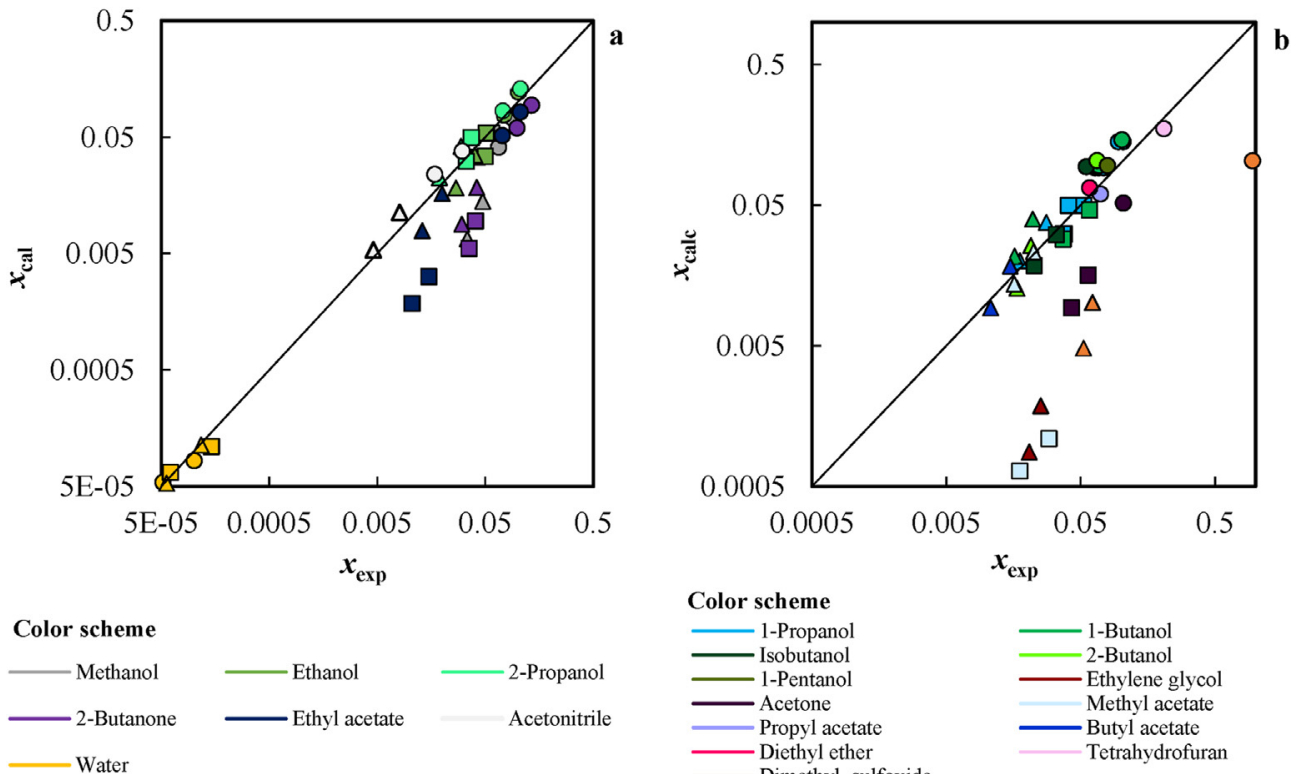

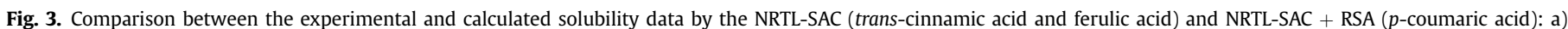
correlation; b) prediction. The symbols correspond to: $\bigcirc$, trans-cinnamic acid; $\square, p$-coumaric acid; $\Delta$, ferulic acid.

Table 5

Estimated solute parameters in the Abraham solvation model, outlier solvent and ARD (\%) for each solute.

\begin{tabular}{|c|c|c|c|c|c|c|c|}
\hline Compound & $E^{\mathrm{a}}$ & $\mathrm{S}$ & A & B & $V^{b}$ & Outlier & ARD (\%) \\
\hline trans-cinnamic acid & 1.301 & 1.100 & 0.482 & 0.479 & 1.171 & methanol & 14 \\
\hline$p$-coumaric acid & 1.582 & 1.781 & 1.143 & 0.546 & 1.229 & ethyl acetate & 26 \\
\hline ferulic acid & 1.472 & 1.138 & 0.290 & 0.877 & 1.429 & ethyl acetate & 18 \\
\hline
\end{tabular}

a Calculated following the procedure described by Abraham et al. [30] using the index of refraction of the solutes obtained from the ACD free software [55].

b Calculated from the molecular structures of the solutes, as described by Abraham [29].

such as alcohols, ketones, esters and nitriles, the parameters obtained in this work are expected to provide reliable solubility predictions for systems where the solute is predominantly present in the monomeric form.

For comparison purposes, the parameters were re-estimated, considering all the solubility data available for trans-cinnamic acid in its monomeric form, in the correlation step. As can be seen in Table S4 of SI, no significant changes were observed either in the parameter values nor in the relative deviations. It shows the robustness of the models, more clearly for the Abraham solvation model, even when using a small number of solvents in the correlation.

In the case of $p$-coumaric acid, Acree and co-authors [40] regressed the Abraham solute descriptors using 32 LFERs, including expressions derived from Eqs. (5-6). The parameters reported by the authors [40] are comparable to those calculated in this work. Despite the broader number of expressions used in the correlation of the parameters, the authors did not include the experimental solubility of $p$-coumaric acid in water, regressing it along with the molar concentration of the solute in the gas phase, obtained from
Eq. (6) and the parameters $S, A$ and $B$. Besides, the value of the parameter $E$ reported by the authors (1.330) is slightly lower than the value estimated in this work (1.582), which also contributes for small differences in the regressed parameters.

The parameters presented in Table 5 were used to estimate the solubilities of the acids in 1-propanol and other organic solvents. An overview of the correlation and prediction results is presented in Fig. 4.

Even if the number of LFERs used in the regression of the solute parameters is reduced, the predicted solubilities are in very good agreement with the experimental solubility data, even for solvents very structurally different, such as propylene glycol (ARD of 21\%) and tetrahydrofuran (ARD of 19\%). The global ARDs obtained were $18 \%$ for trans-cinnamic acid, $26 \%$ for $p$-coumaric acid and $46 \%$ for ferulic acid, being the highest deviations found for the system ferulic acid + butyl acetate.

One of the advantages of the Abraham solvation model is that the descriptors are related to some physicochemical properties of the solute. According to Abraham and co-authors [57], the acidity descriptor $(A)$ represents the strength of $\mathrm{H}$-bonds formed by the 

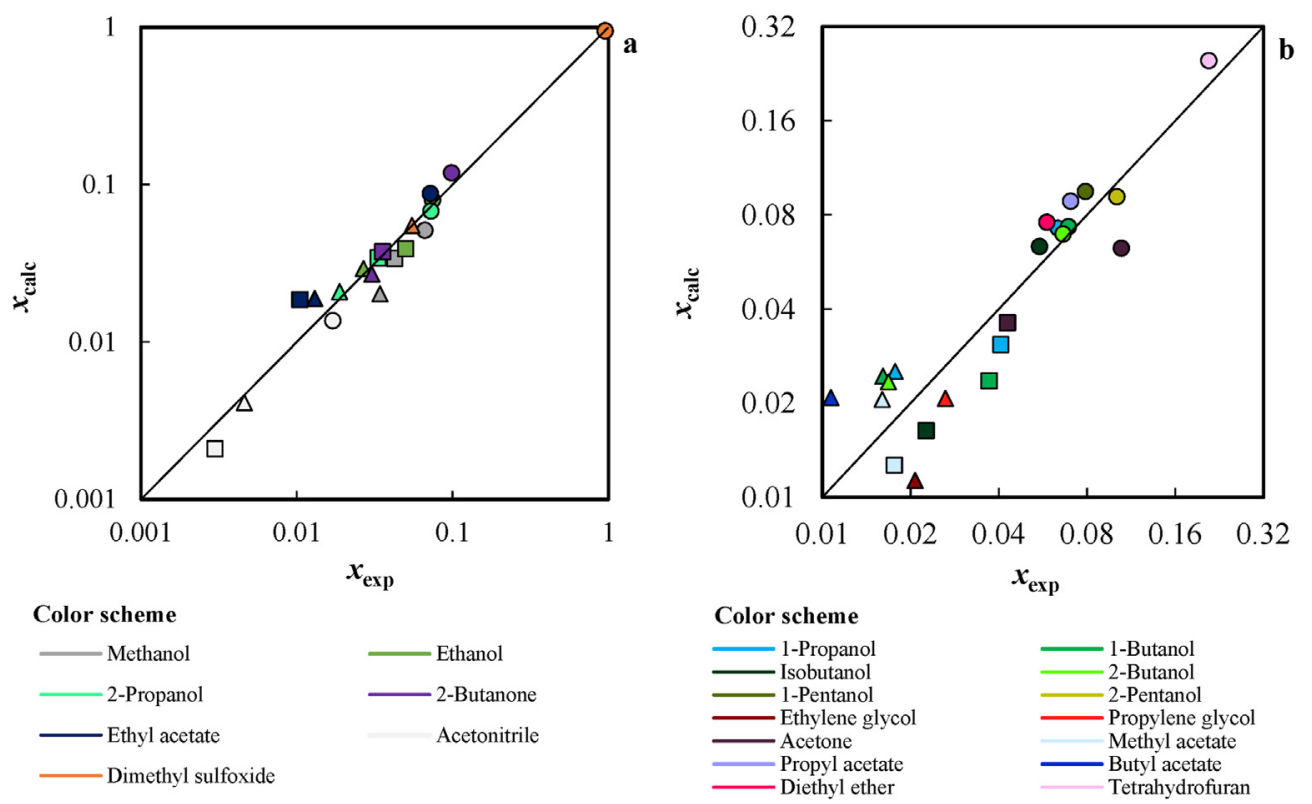

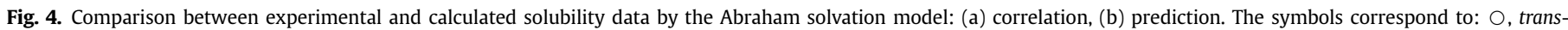
cinnamic acid; $\square, p$-coumaric acid; $\Delta$, ferulic acid.

donor groups when they interact with lone pairs of acceptor groups present in the solvent, whereas the basicity descriptor $(B)$ is related to the strength of the lone pairs of acceptor groups of the solute when there is interaction between the solute and solvents presenting H-bond donor groups. Thus, the presence of hydroxyl groups increases the $\mathrm{H}$-bond acidity of the solute, whereas intramolecular hydrogen bonds tend to reduce it. Looking at the aromatic acids studied in this work, $p$-coumaric acid presents the highest value for the parameter $A$ (1.143), followed by trans-cinnamic acid (0.482) and trans-ferulic acid (0.290). The presence of the hydroxyl group in the para position of the aromatic ring significantly increases the acidity of p-coumaric acid [58] when compared to trans-cinnamic acid, but the presence of the methoxy group in the meta position in trans-ferulic acid probably leads to intramolecular hydrogen bond with the hydroxyl group in the para position, which only partially explains the decrease of the acidity descriptor. Regarding the basicity character, the values of $B$ progressively decrease in the following order: ferulic acid $>p$-coumaric acid > trans-cinnamic acid, which matches very consistently the number of hydrogen acceptors in the molecules.

\section{Conclusions}

In this work, experimental solubility data of three cinnamic acids (trans-cinnamic acid, $p$-coumaric acid and ferulic acid) in water and seven pure organic solvents at $298.2 \mathrm{~K}$ and $313.2 \mathrm{~K}$ are reported. A good agreement with literature was found for all the systems containing trans-cinnamic acid, while some inconsistences were found for the solubility of $p$-coumaric acid and ferulic acid in alcohols, especially at $313.2 \mathrm{~K}$.

The melting temperatures and melting enthalpies of trans-cinnamic acid and ferulic acid were determined by DSC. The melting temperatures of both acids and melting enthalpy of trans-cinnamic acid were in excellent agreement with the literature average value, whereas the melting enthalpy of ferulic acid was slightly higher. The melting properties of $p$-coumaric acid could not be measured due to its decomposition upon melting. The solid phase analysis showed that the solute structure after the evaporation of the solvents corresponds to the structure of the compound as received from the suppliers. The NRTL-SAC was successfully employed to describe the SLE of trans-cinnamic acid and ferulic acid, reaching global ARD of $31 \%$ for the correlations and $42 \%$ for the predictions. Once the melting properties could not be measured for $p$-coumaric acid, the NRTL-SAC model was combined with the RSA to represent the solubility data. Selecting acetonitrile as the reference solvent, the obtained ARDs for the correlation and prediction were $38 \%$ and $40 \%$, respectively.

The Abraham solvation model was also applied to correlate and predict the solubility in organic solvents at $298.2 \mathrm{~K}$. The obtained ARDs obtained in the correlation step for trans-cinnamic acid, $p$ coumaric acid and trans-ferulic acid were $14 \%, 26 \%$ and $18 \%$, respectively, whereas a very satisfactory global ARD for the predictions was found (29\%).

In general, the thermodynamic models used in this work provided an adequate description of the solid-liquid equilibria using a reduced set of experimental solubility data and estimating only a few number of parameters. The ability of estimating solubilities at different temperatures is one of the main advantages of NRTL-SAC, and the model still provides solubility estimates comparable to the experimental data for most of the studied systems. Nevertheless, the Abraham solvation model provides more robust predictions for the solubility of the three aromatic acids at $298.15 \mathrm{~K}$.

\section{CRediT authorship contribution statement}

Sérgio M. Vilas-Boas: Investigation, Writing - original draft, Data curation, Software. Rebeca S. Alves: Investigation. Paula Brandão: Investigation, Data curation, Writing - review \& editing. Leila M.A. Campos: Writing - review \& editing. João A.P. Coutinho: Supervision, Writing - review \& editing. Simão P. Pinho: Supervision, Project administration, Writing - review \& editing. Olga Ferreira: Supervision, Project administration, Writing - review \& editing. 


\section{Declaration of competing interest}

The authors declare that they have no known competing financial interests or personal relationships that could have appeared to influence the work reported in this paper.

\section{Acknowledgments}

This work was developed within the scope of the project CICECO-Aveiro Institute of Materials, UIDB/50011/2020 \& UIDP/ 50011/2020, and CIMO-Mountain Research Center, UIDB/00690/ 2020, both financed by national funds through the Portuguese Foundation for Science and Technology/MCTES. We also acknowledge the support of the projects “AIProcMat@N2020 - Advanced Industrial Processes and Materials for a Sustainable Northern Region of Portugal 2020", with the reference NORTE-01-0145-FEDER000006, supported by Norte Portugal Regional Operational Programme (NORTE 2020), under the Portugal 2020 Partnership Agreement, through the European Regional Development Fund (ERDF); and project AllNat - POCI-01-0145-FEDER-030463, funded by the European Regional Development Fund (ERDF) through the Competitiveness and Internationalization Operational Program (COMPETE2020-POCI) and national funding from the Foundation for Science and Technology (FCT, Portugal). S. M. Vilas-Boas thanks the financial support provided by FCT PhD grant (SFRH/BD/138149/ 2018).

\section{Appendix A. Supplementary data}

Supplementary data to this article can be found online at https://doi.org/10.1016/j.fluid.2020.112747.

\section{References}

[1] L.M.L. Nollet, J.A. Gutierrez-Uribe, Phenolic Compounds in Food: Characterization and Analysis, CRC Press, Boca Raton, 2018.

[2] S. Lafay, A. Gil-Izquierdo, Bioavailability of phenolic acids, Phytochemistry Rev. 7 (2008) 301-311.

[3] M. Sova, Antioxidant and antimicrobial ativities of cinnamic acid derivatives, Mini Rev. Med. Chem. 12 (2012) 749-767.

[4] S.A. Heleno, A. Martins, M.J.R.P. Queiroz, I.C.F.R. Ferreira, Bioactivity of phenolic acids: metabolites versus parent compounds: a review, Food Chem. 173 (2015) 501-513.

[5] F.L. Mota, A.J. Queimada, S.P. Pinho, E.A. Macedo, Aqueous solubility of some natural phenolic compounds, Ind. Eng. Chem. Res. 47 (2008) 5182-5189.

[6] J. Li, Z.X. Zeng, L. Sun, W.L. Xue, H.H. Wang, Solid-liquid phase equilibrium of trans-cinnamic acid in several alcohols: measurements and thermodynamic modeling, J. Chem. Eng. Data 61 (2016) 1192-1198.

[7] A. Noubigh, A. Akremi, Solution thermodynamics of trans-Cinnamic acid in (methanol + water) and (ethanol + water) mixtures at different temperatures, J. Mol. Liq. 274 (2019) 752-758.

[8] J.C. Bradley, M.H. Abraham, W.E. Acree, A.S.I.D. Lang, S.N. Beck, D.A. Bulger, E.A. Clark, L.N. Condron, S.T. Costa, E.M. Curtin, S.B. Kurtu, M.I. Mangir, M.J. McBride, Determination of Abraham model solute descriptors for the monomeric and dimeric forms of trans-cinnamic acid using measured solubilities from the Open Notebook Science Challenge, Chem. Cent. J. 9 (2015) 11, https://doi.org/10.1186/s13065-015-0080-9.

[9] E.I. Alevizou, E.C. Voutsas, Solubilities of p-coumaric and caffeic acid in ionic liquids and organic solvents, J. Chem. Thermodyn. 62 (2013) 69-78.

[10] W. Ji, Q. Meng, P. Li, B. Yang, F. Wang, L. Ding, B. Wang, Measurement and correlation of the solubility of p-coumaric acid in nine pure and water + ethanol mixed solvents at temperatures from 293.15 to $333.15 \mathrm{~K}, \mathrm{~J}$. Chem. Eng. Data 61 (2016) 3457-3465.

[11] C. Zhou, X. Shi, H. Wang, N. An, measurement and correlation of solubilities of adipic acid in different solvents, J. Chem. Ind. Eng. 11 (2007) 2705-2709.

[12] R.G. Bitencourt, F.A. Cabral, A.J.A. Meirelles, Ferulic acid solubility in supercritical carbon dioxide, ethanol and water mixtures, J. Chem. Thermodyn. 103 (2016) 285-291.

[13] N. Haq, N.A. Siddiqui, F. Shakeel, Solubility and molecular interactions of ferulic acid in various (isopropanol + water) mixtures, J. Pharm. Pharmacol. 69 (2017) 1485-1494.

[14] F. Shakeel, M. Salem-Bekhit, N. Haq, N.A. Siddiqui, Solubility and thermodynamics of ferulic acid in different neat solvents: measurement, correlation and molecular interactions, J. Mol. Liq. 236 (2017) 144-150.
[15] F. Shakeel, N. Haq, N.A. Siddiqui, Thermodynamic solubility and solvation behavior of ferulic acid in different (PEG-400 + water) binary solvent mixtures, Drug Dev. Ind. Pharm. 45 (2019) 1468-1476.

[16] H. Wang, Q. Li, W. Deng, E. Omari-Siaw, Q. Wang, S. Wang, S. Wang, X. Cao, X. Xu, J. Yu, Self-nanoemulsifying drug delivery system of trans-cinnamic acid: formulation development and pharmacodynamic evaluation in alloxan-induced type 2 diabetic rat model, Drug Dev. Res. 76 (2015) 82-93.

[17] C.S. Letizia, J. Cocchiara, A. Lapczynski, J. Lalko, A.M. Api, Fragrance material review on cinnamic acid, Food Chem. Toxicol. 43 (2005) 925-943.

[18] K. Pei, J. Ou, J. Huang, S. Ou, p-Coumaric acid and its conjugates: dietary sources, pharmacokinetic properties and biological activities, J. Sci. Food Agric. 96 (2016) 2952-2962.

[19] Y.C. Boo, p-Coumaric acid as an active ingredient in cosmetics: a review focusing on its antimelanogenic effects, Antioxidants 8 (2019) 275.

[20] Z. Zhao, M.H. Moghadasian, Chemistry, natural sources, dietary intake and pharmacokinetic properties of ferulic acid: a review, Food Chem. 109 (2008) 691-702.

[21] S. Ou, K.C. Kwok, Ferulic acid: pharmaceutical functions, preparation and applications in foods, J. Sci. Food Agric. 84 (2004) 1261-1269.

[22] C.-C. Chen, Y. Song, Solubility modeling with NRTL segment activity coefficient model, Ind. Eng. Chem. Res. 43 (2004) 8354-8362.

[23] F.L. Mota, A.P. Carneiro, A.J. Queimada, S.P. Pinho, E.A. Macedo, Temperature and solvent effects in the solubility of some pharmaceutical compounds: measurements and modeling, Eur. J. Pharmaceut. Sci. 37 (2009) 499-507.

[24] F.L. Mota, A.J. Queimada, A.E. Andreatta, S.P. Pinho, E.A. Macedo, Calculation of drug-like molecules solubility using predictive activity coefficient models, Fluid Phase Equil. 322-323 (2012) 48-55.

[25] E. Sheikholeslamzadeh, S. Rohani, Solubility prediction of pharmaceutical and chemical compounds in pure and mixed solvents using predictive models, Ind. Eng. Chem. Res. 51 (2012) 464-473.

[26] Y. Feng, W. Tang, Y. Huang, Y. Xiong, L. Chen, Y. Liu, Y. Li, (Solid + liquid) phase equilibria of tetraphenyl piperazine-1, 4-diyldiphosphonate in pure solvents, J. Chem. Thermodyn. 78 (2014) 143-151.

[27] S.M. Vilas-Boas, P. Brandão, M.A.R. Martins, L.P. Silva, T.B. Schreiner, L. Fernandes, O. Ferreira, S.P. Pinho, Solid phase studies and solubility of isomeric phenolic acids in water and organic solvents, J. Mol. Liq. 272 (2018) 1048-1057.

[28] S.M. Vilas-Boas, V. Vieira, P. Brandão, R.S. Alves, J.A.P. Coutinho, S.P. Pinho, O. Ferreira, Solvent and temperature effects on the solubility of syringic, vanillic or veratric acids: experimental, modeling and solid phase studies, J. Mol. Liq. 289 (2019) 111089.

[29] M.H. Abraham, Scales of solute hydrogen-bonding: their construction and application to physicochemical and biochemical processes, Chem. Soc. Rev. (1992) 73-83, 096.

[30] M.H. Abraham, A. Ibrahim, A.M. Zissimos, Determination of sets of solute descriptors from chromatographic measurements, J. Chromatogr., A 1037 (2004) 29-47.

[31] M.H. Abraham, R.E. Smith, R. Luchtefeld, A.J. Boorem, R. Luo, W.E. Acree Jr., Prediction of solubility of drugs and other compounds in organic solvents, J. Pharm. Sci. 99 (2010) 1500-1515.

[32] K.R. Hoover, D.M. Stovall, E. Pustejovsky, R. Coaxum, K. Pop, W.E. Acree Jr., M.H. Abraham, Solubility of crystalline nonelectrolyte solutes in organic solvents: mathematical correlation of 2-methoxybenzoic acid and 4methoxybenzoic acid solubilities with the Abraham solvation parameter model, Can. J. Chem. 82 (2004) 1353-1360.

[33] D.M. Stoval, C. Givens, S. Keown, K.R. Hoover, R. Barnes, C. Harris, J. Lozano, M. Nguyen, E. Rodriguez, W.E. Acree Jr., M.H. Abraham, Solubility of crystalline nonelectrolyte solutes in organic solvents: mathematical correlation of 4chloro-3-nitrobenzoic acid and 2-chloro-5- nitrobenzoic acid solubilities with the Abraham solvation parameter model, Phys. Chem. Liq. 43 (2005) $351-360$.

[34] C.R. Daniels, A.K. Charlton, R.M. Wold, W.E. Acree Jr., M.H. Abraham, Thermochemical behavior of dissolved carboxylic acid solutes: solubilities of 3methylbenzoic acid and 4-chlorobenzoic acid in organic solvents, Can. J. Chem. 81 (2003) 1492-1501.

[35] A.K. Charlton, C.R. Daniels, R.M. Wold, E. Pustejovsky, W.E. Acree, M.H. Abraham, Solubility of crystalline nonelectrolyte solutes in organic solvents: mathematical correlation of 3-nitrobenzoic acid solubilities with the Abraham general solvation model, J. Mol. Liq. 116 (2005) 19-28.

[36] S. Ye, M. Saifullah, L.M. Grubbs, M.C. McMillan-Wiggins, P. Acosta, D. Mejorado, I. Flores, W.E. Acree, M.H. Abraham, Determination of the Abraham model solute descriptors for 3,5-dinitro-2-methylbenzoic acid from measured solubility data in organic solvents, Phys. Chem. Liq. 49 (2011) $821-829$.

[37] W.E. Acree, K.R. Bowen, M.Y. Horton, M.H. Abraham, Computation of Abraham model solute descriptors for 3-methyl-4-nitrobenzoic acid from measured solubility data, Phys. Chem. Liq. 55 (2017) 482-491.

[38] K.R. Hoover, R. Coaxum, E. Pustejovsky, D.M. Stovall, W.E. Acree, M.H. Abraham, Thermochemical behavior of dissolved carboxylic acid solutes: part 4 - mathematical correlation of 4-nitrobenzoic acid solubilities with the abraham solvation parameter model, Phys. Chem. Liq. 42 (2004) 339-347.

[39] K.R. Hoover, K. Pop, W.E. Acree Jr., M.H. Abraham, Solubility of crystalline nonelectrolyte solutes in organic solvents: mathematical correlation of 3chlorobenzoic acid solubilities with the Abraham solvation parameter model, S. Afr. J. Chem. 58 (2005) 25-29. 
[40] W.E. Acree, M. Barrera, M.H. Abraham, Comment on "measurement and correlation of the solubility of p-coumaric acid in nine pure and water + ethanol mixed solvents at temperatures from 293.15 to 333.15 K, J. Chem. Eng. Data 62 (2017) 578-583.

[41] O. Ferreira, S. Pinho, Solubility of flavonoids in pure solvents, Ind. Eng. Chem. Res. 51 (2012) 6586-6590.

[42] C.-C. Chen, P.A. Crafts, Correlation and prediction of drug molecule solubility with the NRTL-SAC model, Comput. Aided Chem. Eng. 21 (2006) 859-864.

[43] S. Vilas-Boas, P. Brandão, M.A.R. Martins, L.P. Silva, L. Fernandes, O. Ferreira, S.P. Pinho, Solid Phase Studies and Solubility of isomeric phenolic acids in water and organic solvents, in: Int. Symp. Solubility Phenom, Tours, 2018, p. 8362.

[44] J.M. Prausnitz, R.N. Lichtenthaler, E.G. de Azevedo, Molecular Thermodynamics of Fluid-phase Equilibria, Prentice Hall PTR, 1999.

[45] J. Abildskov, J.P. O'Connell, Predicting the solubilities of complex chemicals I. Solutes in different solvents, Ind. Eng. Chem. Res. 42 (2003) 5622-5634.

[46] J. Abildskov, J.P. O'Connell, Thermodynamic method for obtaning the solubilities of complex medium-sized chemicals in pure and mixed solvents, Fluid Phase Equil. 228-229 (2005) 395-400.

[47] V.N. Emel'yanenko, A.V. Yermalayeu, M. Voges, C. Held, G. Sadowski, S.P. Verevkin, Thermodynamics of a model biological reaction: a comprehensive combined experimental and theoretical study, Fluid Phase Equil. 422 (2016) 99-110.

[48] W.E. Acree, Thermodynamic properties of organic compounds: enthalpy of fusion and melting point temperature compilation, Thermochim. Acta 189 (1991) 37-56.

[49] L. Sturz, V.T. Witusiewicz, U. Hecht, S. Rex, Organic alloy systems suitable for the investigation of regular binary and ternary eutectic growth, J. Cryst.
Growth 270 (2004) 273-282.

[50] B.L. Sharma, R. Jamwal, R. Kant, Thermodynamic and lamella models relationship for the eutectic system benzoic acid - cinnamic acid, Cryst. Res. Technol. 39 (2004) 454-464.

[51] M.S. Manic, D. Villanueva, T. Fornari, A.J. Queimada, E.A. Macedo, V. Najdanovic-Visak, Solubility of high-value compounds in ethyl lactate: measurements and modeling, J. Chem. Thermodyn. 48 (2012) 93-100.

[52] J.Z. Dávalos, R. Herrero, A. Chana, A. Guerrero, P. Jiménez, J.M. Santiuste, Energetics and structural properties, in the gas phase, of trans -hydroxycinnamic acids, J. Phys. Chem. 116 (2012) 2261-2267.

[53] A. Noubigh, M. Abderrabba, E. Provost, Temperature and salt addition effects on the solubility behaviour of some phenolic compounds in water, J. Chem. Thermodyn. 39 (2007) 297-303.

[54] E. Königsberger, Editorial: guidelines for the measurement of solid-liquid solubility data at atmospheric pressure, J. Chem. Eng. Data 64 (2019) 381-385.

[55] I. (ACD/Labs), Advanced Chemistry Development, Percepta Predictors, 2017.

[56] J.A. Platts, D. Butina, M.H. Abraham, A. Hersey, Estimation of molecular linear free energy relation descriptors using a group contribution approach, J. Chem. Inf. Comput. Sci. 39 (1999) 835-845.

[57] M.H. Abraham, A. Ibrahim, A.M. Zissimos, Y.H. Zhao, J. Comer, D.P. Reynolds Application of hydrogen bonding calculations in property based drug design, Drug Discov. Today 7 (2002) 1056-1063.

[58] D.O. Abranches, M.A.R. Martins, L.P. Silva, N. Schaeffer, S.P. Pinho, J.A.P. Coutinho, Phenolic hydrogen bond donors in the formation of non-ionic deep eutectic solvents: the quest for type v DES, Chem. Commun. 55 (2019) 10253-10256. 